\title{
Tumor Growth Control by TP-LPV-LMI based Controller
}

\author{
György Eigner \\ Dániel András Drexler \\ Levente Kovács \\ Physiological Controls Research Center Physiological Controls Research Center Physiological Controls Research Center \\ Research, Innovation and Service Center Research, Innovation and Service Center Research, Innovation and Service Center \\ of Óbuda University Center \\ of Óbuda University Center \\ of Óbuda University Center \\ Budapest, Hungary +36-70-391-5853 Budapest, Hungary +36-1-666-5530 \\ Budapest, Hungary +36-1-666-5585 \\ Email: eigner.gyorgy@nik.uni-obuda.hu Email: drexler.daniel@nik.uni-obuda.hu Email: kovacs.levente@nik.uni-obuda.hu
}

\begin{abstract}
The advantages of using advanced control techniques related to physiological applications are unquestionable as it was proven in many cases in the recent times. Although, there are several challenges that practitioners need to face. For example, the lack of precise information about the internal state of the patients, i.e. the inter- and intra-patient variabilities which cause uncertainties that need to be tolerated by the applied controllers. In this study an alternative solution is presented for control of tumor growth. Uncertainties and nonlinearities are handled by the applied Linear Parameter Varying (LPV) methodology completed by Tensor Product (TP) model transformation. Linear Matrix Inequalities (LMI) based optimization are used for controller design. The lack of information about the internal state is solved by using Extended Kalman Filter (EKF) to estimate the non-measurable state variables. The developed control structure is able to enforce the controlled system to behave as a predefined reference system. We show that the control framework operates well and reaches the determined aims of the control.

Index Terms-Tensor Model transformation, Linear Parameter Varying, Linear Matrix Inequality, Parallel Distribution Control, tumor control
\end{abstract}

\section{INTRODUCTION}

In this study we investigate the usability of Targeted Molecular Therapy (TMT) in case of control of tumor growth from control engineering point of view with respect to modern control techniques. TMT is an innovative treatment option for patients suffering from cancer with many advantages, e.g. the side effects are less harmful and the targeting is more specific compared to regular interventions such as chemotherapy or radiotherapy [1], [2]. TMTs exert their effect by blocking some specific properties of the tumors.

A widely used drug type are the angiogenic inhibitors [3], which are able to interfere with the formation of new blood vessels. From the tumor concourses' viewpoint that means they are not able to growth after a certain limit and their volume decreases due to the phenomena that after the diffusion barrier the tumor concourses need own blood vessels to get nutrients from the blood in order to supply their growing [4]. One of

Gy. Eigner was supported by the ÚNKP-17-4/I. New National Excellence Program of the Ministry of Human Capacities. This project has received funding from the European Research Council (ERC) under the European Union's Horizon 2020 research and innovation programme (grant agreement No 679681) the applied drug is endostatin, which was taken into account in this study as well.

In our former work we have proven that the tumor growth inhibition can be formulated as a control problem and we provided optimal and nonlinear solutions for control of tumor growth [5]-[8]. In this study an alternative control approach is introduced by facilitating the LPV framework [9], [10], LMI optimization [11], [12], TP model transformation [13]-[16] and mixed EKF design [17].

The paper is structured as follows. First, the applied tumor growth model and the developed qLPV model are introduced in Section II. The controller design steps are presented in Section III. The results are shown in Section IV, and the paper ends with the conclusions in Section V.

\section{Investigated Tumor Growth Model}

In this study we have examined a modified version of the extended Hahnfeldt-model [7], [8], [18] which considers the dynamics of the inhibitor intake as well - described by (3). The applied modified version of the given model appeared in [8].

The extended Hahnfeldt-model consists of the following differential equations [7]:

$$
\begin{gathered}
\dot{z}_{1}(t)=-\lambda_{1} z_{1}(t) \ln \left(\frac{z_{1}(t)}{z_{2}(t)}\right), \\
\dot{z}_{2}(t)=b z_{1}(t)-d z_{1}^{2 / 3}(t) z_{2}(t)-\eta z_{2}(t) z_{3}(t), \\
\dot{z_{3}}(t)=-\lambda_{3} z_{3}(t)+u(t) .
\end{gathered}
$$

The state variables are the tumor volume $z_{1}(t)\left[\mathrm{mm}^{3}\right]$, volume of supporting vasculature $z_{2}(t)\left[\mathrm{mm}^{3}\right]$ and the inhibitor serum level $z_{3}(t)[\mathrm{mg} / \mathrm{kg}]$. The output of the model is the tumor volume $z_{1}(t)$, which is considered as measurable. The parameters applied in this study are the following: $\lambda_{1}=0.1921$ $1 /$ day, $b=5.8511 /$ day, $d=0.008711 /\left(\mathrm{mm}^{2}\right.$ day $), \eta=0.66$ $\mathrm{kg} /\left(\mathrm{mg}\right.$ day) based on [18]. The $\lambda_{3}=1.31$ 1/day clearence rate belongs to the assumed inhibitor (endostatin) [18].

The model does have crucial limitations. The main ones are the feasibility and numerical stability issues which may occur 
when $z_{1}$ and $z_{2}$ are tending to 0 in $\ln \left(\frac{z_{1}(t)}{z_{2}(t)}\right)$ term of (1), namely, approaching the $(0 / 0)$ type singularity.

By introducing new - transformed - state variables in accordance with [8], [19], the model can be transformed into a more suitable form. Let the new state variables be the following: $x_{1}(t)=\ln \left(z_{1}(t)\right), x_{2}(t)=\ln \left(z_{2}(t)\right)$, and $x_{3}(t)=z_{3}(t)$. This consideration leads to the following, transformed extended Hahnfeldt-model [8]:

$$
\begin{gathered}
\dot{x}_{1}(t)=-\lambda_{1} x_{1}(t)+\lambda_{1} x_{2}(t), \\
\dot{x}_{2}(t)=b e^{x_{1}(t)-x_{2}(t)}-d e^{2 x_{1}(t) / 3}-\eta x_{3}(t), \\
\dot{x}_{3}(t)=-\lambda_{3} x_{3}(t)+u(t) .
\end{gathered}
$$

The $z_{1}$ and $z_{2}$ state variables are limited, i.e. the nontrivial equilibrium of the model can be calculated based on (1)(3) beside permanent inhibitor level $\left(z_{3}(t) \equiv z_{3, \infty}\right)$ in the following way [7], [20]:

$$
\begin{aligned}
& z_{1, \infty}=z_{2, \infty}=\left(\frac{b-\eta z_{3, \infty}}{d}\right)^{3 / 2} \\
& z_{1, \max }=z_{2, \max }=\left(\frac{b}{d}\right)^{3 / 2} \leftrightarrow z_{3, \infty}=\frac{1}{\lambda_{3}} u_{\infty} \equiv 0 .
\end{aligned}
$$

Equation (7) shows that the operating domain of the $z_{1}$ and $z_{2}$ original state variables are $z_{1}(t), z_{2} \in\left(0,(b / d)^{3 / 2}\right]$. We suppose, that the original state variables has a lower limit of 1 $\mathrm{mm}^{3}$, thus the the transformed state variables are in the domain $x_{1}, x_{2} \in\left(0, \ln \left((b / d)^{3 / 2}\right)\right]$. In accordance with [8], the goal of the control can be determined as $x_{1}=x_{2}=0$.

\section{A. qLPV model development}

The LPV models can be formulated in state-space form in the following way [9], [21]:

$$
\begin{gathered}
\dot{\mathbf{x}}(t)=\mathbf{A}(\mathbf{p}(t)) \mathbf{x}(t)+\mathbf{B}(\mathbf{p}(t)) \mathbf{u}(t) \\
\mathbf{y}(t)=\mathbf{C}(\mathbf{p}(t)) \mathbf{x}(t)+\mathbf{D}(\mathbf{p}(t)) \mathbf{u}(t) \\
\left(\begin{array}{l}
\dot{\mathbf{x}}(t) \\
\mathbf{y}(t)
\end{array}\right)=\mathbf{S}(\mathbf{p}(t))\left(\begin{array}{l}
\mathbf{x}(t) \\
\mathbf{u}(t)
\end{array}\right) \\
\mathbf{S}(\mathbf{p}(t))=\left[\begin{array}{ll}
\mathbf{A}(\mathbf{p}(t)) & \mathbf{B}(\mathbf{p}(t)) \\
\mathbf{C}(\mathbf{p}(t)) & \mathbf{D}(\mathbf{p}(t))
\end{array}\right]
\end{gathered}
$$

$\mathbf{A}(\mathbf{p}(t)) \in \mathbb{R}^{n \times n}, \mathbf{B}(\mathbf{p}(t)) \in \mathbb{R}^{n \times m}, \mathbf{C}(\mathbf{p}(t)) \in \mathbb{R}^{k \times n}$, $\mathbf{D}(\mathbf{p}(t)) \in \mathbb{R}^{k \times m}$ and $\mathbf{S}(\mathbf{p}(t)) \in \mathbb{R}^{n+k \times n+m}$ are the $\mathbf{p}(t)$ dependent state, input, output, feed-forward and system matrices, respectively. $\mathbf{x}(t) \in \mathbb{R}^{n}, \mathbf{y}(t) \in \mathbb{R}^{k}$ and $\mathbf{u}(t) \in \mathbb{R}^{m}$ are the state, output and input vectors, respectively. The $\mathbf{p}(t)=\left[p_{1}(t) \ldots p_{R}(t)\right]$ parameter vector consists of the scheduling parameters $p_{i}(t)$. In accordance with the LPV theorems [9], the $\mathbf{p}(t) \in \mathbf{\Omega}^{R} \in \mathbb{R}^{R}$ is an $R$-dimensional real vector within the $\boldsymbol{\Omega}=\left[p_{1, \min }, p_{1, \max }\right] \times\left[p_{2, \min }, p_{2, \max }\right] \times$ $\ldots \times\left[p_{R, \text { min }}, p_{R, \max }\right] \in \mathbb{R}^{R}$ hyperspace inward the $\mathbb{R}^{R}$ real vector space. A quasi-LPV (qLPV) model obtains if any of the state variables are involved into the parameter vector [9].

By encapsulating the nonlinearity causing terms from (4)(6) into $\mathbf{p}(t)$ in accordance with the LPV methodology we are able to use linear controller design techniques and the resulting
LPV controller is able to handle the original nonlinear system as well [9].

We have developed an error dynamics based control oriented qLPV model [13], [15], [22] for control purposes. The model is able to describe the deviation between the state variables of the system to be controlled and a prescribed equilibrium or a reference system. Namely, we have introduced transformed state variables and input: $\Delta \mathbf{x}(t)=\mathbf{x}(t)-\mathbf{x}_{r e f}(t)$ and $\Delta u(t)=u(t)-u_{r e f}(t)$. By applying state-feedback controller, the control goal becomes to eliminate $\Delta \mathbf{x}(t)$ over time, namely, $\Delta \mathbf{x}(t) \rightarrow \mathbf{0}, t \rightarrow \infty$.

The transformation of the first and third states is straightforward, i.e.:

$$
\begin{aligned}
& \Delta \dot{x}_{1}(t)=\dot{x}_{1}(t)-\dot{x}_{1, \text { ref }}(t)= \\
& -\lambda_{1} x_{1}(t)+\lambda_{1} x_{2}(t)- \\
& \left(-\lambda_{1} x_{1, \text { ref }}(t)+\lambda_{1} x_{2, \text { ref }}(t)\right)= \\
& -\lambda_{1}\left(x_{1}(t)-x_{1, \text { ref }}(t)\right)+\lambda_{1}\left(x_{2}(t)-x_{2, \text { ref }}(t)\right)= \\
& -\lambda_{1} \Delta x_{1}(t)+\lambda_{1} \Delta x_{2}(t) . \\
& \Delta \dot{x}_{3}(t)=\dot{x}_{3}(t)-\dot{x}_{3, \text { ref }}(t)= \\
& -\lambda_{3} x_{3}(t)+u(t)-\left(-\lambda_{3} x_{3, \text { ref }}(t)+u_{\text {ref }}(t)\right)= \\
& -\lambda_{3} \Delta x_{3}(t)+\Delta u(t) .
\end{aligned}
$$

However, the transformation of the second state is not trivial.

$$
\begin{aligned}
& \Delta \dot{x}_{2}(t)=\dot{x}_{2}(t)-\dot{x}_{2, r e f}(t)= \\
& b e^{x_{1}(t)-x_{2}(t)}-d e^{2 x_{1}(t) / 3}-\eta x_{3}(t) \\
& -\left(b e^{x_{1, r e f}(t)-x_{2, r e f}(t)}-d e^{2 x_{1, r e f}(t) / 3}-\eta x_{3, \text { ref }}(t)\right) .
\end{aligned}
$$

By investigating the terms belonging together from (10), the following mathematical manipulations can be done:

$$
\begin{aligned}
& b e^{x_{1}(t)} e^{-x_{2}(t)}-b e^{x_{1, r e f}(t)} e^{-x_{2, r e f}(t)}-0= \\
& b e^{x_{1}(t)} e^{-x_{2}(t)}-b e^{x_{1, r e f}(t)} e^{-x_{2, r e f}(t)}-b e^{x_{1, r e f}(t)} e^{-x_{2}(t)} \\
& +b e^{x_{1, r e f}(t)} e^{-x_{2}(t)}= \\
& b e^{-x_{2}(t)}\left(e^{x_{1}(t)}-e^{x_{1, r e f}(t)}\right) \cdot 1 \\
& -b e^{x_{1, r e f}(t)}\left(e^{-x_{2}(t)}-e^{-x_{2, r e f}(t)}\right) \cdot 1= \\
& b e^{-x_{2}(t)} \frac{\left(e^{x_{1}(t)}-e^{x_{1, r e f}(t)}\right)}{\Delta x_{1}(t)} \Delta x_{1}(t) \\
& -b e^{x_{1, r e f}(t)} \frac{\left(e^{-x_{2}(t)}-e^{-x_{2, r e f}(t)}\right)}{\Delta x_{2}(t)} \Delta x_{2}(t) \\
& -d e^{2 x_{1}(t) / 3}+d e^{2 x_{1, r e f}(t) / 3}= \\
& -d\left(e^{2 x_{1}(t) / 3}-e^{2 x_{1, r e f}(t) / 3}\right) \cdot 1= \\
& -d \frac{\left(e^{2 x_{1}(t) / 3}-e^{2 x_{1, r e f}(t) / 3}\right)}{\Delta x_{1}(t)} \Delta x_{1}(t) . \\
& -\eta x_{3}(t)+\eta x_{3, r e f}(t)=-\eta \Delta x_{3}(t) .
\end{aligned}
$$

From (12), two scheduling variables can be selected: $p_{1}(t)=b e^{-x_{2}(t)} \frac{\left(e^{x_{1}(t)}-e^{x_{1}, r e f}(t)\right.}{\Delta x_{1}(t)}-d \frac{\left(e^{2 x_{1}(t) / 3}-e^{2 x_{1, r e f}(t) / 3}\right)}{\Delta x_{1}(t)}$ and $p_{2}(t)=-b e^{x_{1, r e f}(t)} \frac{\left(e^{-x_{2}(t)}-e^{-x_{2}, r e f(t)}\right)}{\Delta x_{2}(t)}$. In this way, the transformed $\Delta x_{2}(t)$ state becomes as follows:

$$
\Delta \dot{x}_{2}(t)=p_{1}(t) \Delta x_{1}(t)+p_{2}(t) \Delta x_{2}(t)-\eta \Delta x_{3}(t) .
$$

It should be noted that the $p_{1}$ and $p_{2}$ may cause numerical instability, when $\Delta x_{1}, \Delta x_{2} \rightarrow 0$. By applying the L'Hospital's 
rule [23], we get that both terms have finite final values which allow the application of them in practice without the mentioned stability issues.

$$
\begin{gathered}
\lim _{\Delta x_{1}(t) \rightarrow 0} b e^{-x_{2}(t)} \frac{\left(e^{x_{1}(t)}-e^{x_{1, r e f}(t)}\right)}{\Delta x_{1}(t)} \\
-d \frac{\left(e^{2 x_{1}(t) / 3}-e^{2 x_{1, r e f}(t) / 3}\right)}{\Delta x_{1}(t)}= \\
\left(e^{x_{1}(t)}+e^{x_{1} r e f(t)}\right)\left(\frac{2}{3} d+b e^{2 x_{2}(t)}\right) \cdot \\
\lim _{\Delta x_{2}(t) \rightarrow 0} b e^{-x_{1, r e f}(t)} \frac{\left(e^{x_{2}(t)}-e^{x_{2, r e f}(t)}\right)}{\Delta x_{2}(t)}= \\
-b e^{x_{r e f, 1}(t)}\left(e^{x_{2}(t)}+e^{x_{2, r e f}(t)}\right) .
\end{gathered}
$$

The domains of $p_{1}$ and $p_{2}$ are $p_{1}(t)=[0, \ldots, 13]$ and $p_{2}(t)=[4, \ldots, 15]$. These domains are acquired from the domains of $x_{1}$ and $x_{2}$.

Hence, the following LPV form is obtained by considering (9), (10) and (13):

$$
\begin{gathered}
\dot{\mathbf{x}}(t)=\mathbf{A}(\mathbf{p}(t)) \mathbf{x}(t)+\mathbf{B u}(t) \\
\mathbf{y}(t)=\mathbf{C} \mathbf{x}(t) \\
\mathbf{S}(\mathbf{p}(t))=\left[\begin{array}{cc}
\mathbf{A}(\mathbf{p}(t)) & \mathbf{B} \\
\mathbf{C} & 0
\end{array}\right]=\left[\begin{array}{cccc}
-\lambda_{1} & \lambda_{1} & 0 & 0 \\
p_{1}(t) & p_{2}(t) & -\eta & 0 \\
0 & 0 & -\lambda_{3} & 1 \\
1 & 0 & 0 & 0
\end{array}\right] .
\end{gathered}
$$

\section{Controller Design}

\section{A. TP Model and Controller}

By applying TP model transformation a qLPV function represented by $\mathbf{S}(\mathbf{p}(t))$ - can be transformed into a finite element convex polytopic TP model.

$$
\begin{gathered}
\left(\begin{array}{c}
\dot{\mathbf{x}}(t) \\
\mathbf{y}(t)
\end{array}\right)=\mathbf{S}(\mathbf{p}(t))\left(\begin{array}{c}
\mathbf{x}(t) \\
\mathbf{u}(t)
\end{array}\right) \\
\mathbf{S}(\mathbf{p}(t))=\mathcal{S} \underset{\mathrm{r}=1}{\mathbb{\mathrm { R }}} \mathbf{w}_{r}\left(p_{r}(t)\right)=\mathcal{S} \times_{r} \mathbf{w}(\mathbf{p}(t)) .
\end{gathered}
$$

The $\mathcal{S} \in \mathbb{R}^{I_{1} \times I_{2} \times \ldots \times I_{R} \times(n+k) \times(n+m)}$ is the core tensor, $\mathbf{S}_{i_{1}, i_{2}, \ldots, i_{R}}$ are linear time invariant (LTI) system vertices, $\mathbf{w}_{r}\left(p_{r}(t)\right)$ is the weighting vector, and $w_{r, i_{r}}\left(p_{r}(t)\right)$ $\left(i_{r}=1 \ldots I_{R}\right)$ are the continuous convex weighting functions. By considering that $\forall r, i, p_{r}(t): w_{r, i_{r}}\left(p_{r}(t)\right) \in[0,1]$ and $\forall r, p_{r}(t): \sum_{i=1}^{I_{r}} w_{r, i_{r}}\left(p_{r}(t)\right)=1$, the convexity can be held. From the available convex hulls we have applied the Minimal Volume Simplex (MVS) type convex hull in this study [24]. The execution steps of the TP model transformation are available in [13], [15], [22], [25]-[27].

An LPV based general state-feedback controller can be realized as follows:

$$
\mathbf{u}(t)=\mathbf{r}(t)-\mathbf{G}(\mathbf{p}(t)) \mathbf{x}(t),
$$

where $\mathbf{G}(\mathbf{p}(t)) \in \mathbb{R}^{m \times n}$ is the p-dependent controller gain matrix. By applying $\mathbf{r}(t)=\mathbf{0}^{n \times 1}$ reference, (17) simplifies to $\mathbf{u}(t)=-\mathbf{G}(\mathbf{p}(t)) \mathbf{x}(t)$. The finite element convex TP controller based on polytopic structure is the following:

$$
\mathbf{G}(\mathbf{p}(t))=\mathcal{G} \underset{\mathrm{r}=1}{\mathrm{R}} \mathbf{w}_{r}\left(p_{r}(t)\right)=\mathcal{G} \times_{r} \mathbf{w}(\mathbf{p}(t))
$$

The $\mathcal{G}$ controller gain tensor consists of the $\mathbf{G}_{i_{1}, i_{2}, \ldots, i_{R}}$ feedback gain vertices (matrices). Each of them belongs to a certain $\mathbf{S}_{i_{1}, i_{2}, \ldots, i_{R}}$ LTI system structure. The weighting function $\mathbf{w}_{r}\left(p_{r}(t)\right)$ is the same as in (16). Namely, the $\mathcal{S}$ and $\mathcal{G}$ are connected via $\mathbf{w}_{r}\left(p_{r}(t)\right)$. The resulting TP controller $\mathcal{G}(\mathbf{p}(t))$ is able to handle via the state-feedback the $\mathbf{S}(\mathbf{p}(t))$ TP model and the original nonlinear system as well.

\section{B. LMI based Controller Design}

It is possible to define convex constraints as LMIs regarding the controller design related to convex TP model structures. Namely, the LMI based controller design can be done via numerical optimization of convex objective functions with respect to the developed qLPV based TP model. The obtained controller is able to keep these constraints during operation.

We have applied the Parallel Distributed Compensation (PDC) framework through which a quadratically stabilizing state-feedback type PDC controller can be designed for continuous polytopic systems by using LMI optimization. A possible solution to develop PDC type controller via LMI optimization is the design of LMI regions through pole clustering. Pole clustering allows us to determine the place of the poles of the closed system in the complex plane.

A $\mathcal{D}$ region in the complex plane is an LMI region if there exists an $\alpha=\left[\alpha_{i j}\right] \in \mathbb{R}^{q \times q}$ symmetric matrix and $\beta=\left[\beta_{i j}\right] \in$ $\mathbb{R}^{c \times q}$ matrix such that $\mathcal{D}:=\left\{z \in \mathbb{C}: f_{\mathcal{D}}(z)=\alpha+\beta z+\beta^{\top} \bar{z}<\right.$ $0\}$ [28]. Let the $\mathcal{D}$ region be in the negative half-plane of the complex plane. The $\dot{x}(t)=\mathbf{A} x(t)$ dynamical system is $\mathcal{D}$ stable if all of its poles lie in the region $\mathcal{D}$ [28]. $\mathbf{A}$ is $\mathcal{D}$-stable iff there exists a symmetric positive definite $\mathbf{X}>0$ matrix such that $M_{\mathcal{D}}(\mathbf{A}, \mathbf{X}):=\alpha \otimes \mathbf{X}+\beta \otimes \mathbf{A X}+(\beta \otimes \mathbf{A X})^{\top}<0$ where $\otimes$ is the Kronecker-product. The connection between $f_{\mathcal{D}}(z)$ and $M_{\mathcal{D}}(\mathbf{A}, \mathbf{X})$ is that $(1, z, \bar{z}) \leftrightarrow\left(\mathbf{X}, \mathbf{A X}, \mathbf{X A}^{\top}\right)$ [28], [29]. In conformity with [11], [12], [28], [30] it is possible to design a PDC type state feedback controller via appropriate $\mathbf{G}(\mathbf{p}(t))$ controller gains, which is able to provide the $\mathcal{D}$ stability and enforces the poles of the closed system to lie in the determined (negative) part of the complex plane. To facilitate this idea the closed-loop system has to be considered during the controller design: $\left.(\mathbf{A X}),(\mathbf{A X})^{\top} \leftrightarrow(\mathbf{A X}+\mathbf{B M}),(\mathbf{A X}+\mathbf{B M})^{\top}\right)$, where $\mathbf{M}$ is a matrix variable and $\mathbf{G}$ can be calculated as $\mathbf{G}:=\mathbf{M X}^{-1}$.

We have considered two LMIs in order to characterize the distribution of the poles of the closed system, the so-called $\alpha$ stability and disk region LMIs. These are the following:

$$
(\mathbf{X A}+\mathbf{B M})+(\mathbf{X A}+\mathbf{B M})^{\top}+2 \alpha \mathbf{X}<0
$$

and

$$
\left[\begin{array}{cc}
-r \mathbf{X} & -q \mathbf{X}+(\mathbf{X A}+\mathbf{B M}) \\
-q \mathbf{X}+(\mathbf{X A}+\mathbf{B M})^{\top} & -r \mathbf{X}
\end{array}\right]<0,
$$


where $\alpha$ determines a boundary from which all of the poles lie leftward, while $r$ (radius) and $q$ (center) determine a disk in which the poles fall into. Thus, the overall shape of the $\mathcal{D}$ region becomes a half-disk (Fig. 1). The $\mathbf{G}_{i}$ gains are obtained

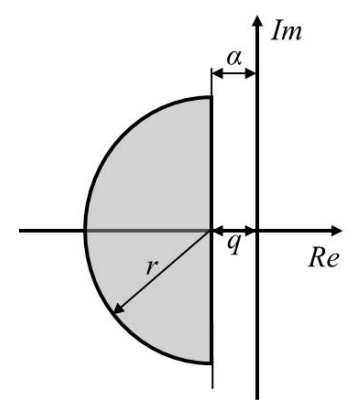

Figure 1. $\lambda(\mathbf{A}+\mathbf{B G})$ poles of the closed system inside the $\mathcal{D}$ complex region.

after solving the feasibility-kind LMI optimization problem to which the YALMIP framework [31] and the SeDuMi 1.3 solver have been applied [32] with respect to the difference based qLPV model from (15):

Subjects : $\mathbf{X}, \mathbf{M}$

$\mathbf{X}>0$,

$$
\begin{aligned}
& \left(\mathbf{X A}_{i}+\mathbf{B M}_{i}\right)+\left(\mathbf{X} \mathbf{A}_{i}+\mathbf{B M}_{i}\right)^{\top}+2 \alpha \mathbf{X}<0, \\
& \left(\mathbf{X} \mathbf{A}_{i}+\mathbf{B M}_{j}\right)+\left(\mathbf{X} \mathbf{A}_{i}+\mathbf{B M}_{j}\right)^{\top}+2 \alpha \mathbf{X}<0, \\
& {\left[\begin{array}{cc}
-r \mathbf{X} & -q \mathbf{X}+\left(\mathbf{X A}_{i}+\mathbf{B M}_{i}\right) \\
-q \mathbf{X}+\left(\mathbf{X} \mathbf{A}_{i}+\mathbf{B M}_{i}\right)^{\top} & -r \mathbf{X}
\end{array}\right]<0,} \\
& {\left[\begin{array}{cc}
-r \mathbf{X} & -q \mathbf{X}+\left(\mathbf{X} \mathbf{A}_{i}+\mathbf{B M}_{j}\right) \\
-q \mathbf{X}+\left(\mathbf{X} \mathbf{A}_{i}+\mathbf{B M}_{j}\right)^{\top} & -r \mathbf{X}
\end{array}\right]<0,} \\
& i<j \leq R \text { s.t. } \forall \mathbf{p}(t): w_{i}(\mathbf{p}(t)) w_{j}(\mathbf{p}(t))=0,
\end{aligned}
$$

in which we have considered that $\alpha=0, q=0$ and $r=12$ in order to avoid the too "fast" poles which may lead to drastic interventions into the control process via the control signal. The obtained $\mathbf{G}_{1, \ldots, 4}$ gains are the following: $\mathbf{G}_{1}=\left[\begin{array}{lll}225.025 & 292.6693 & -22.0255\end{array}\right], \mathbf{G}_{2}=$ $\left[\begin{array}{ll}585.19 & 283.3383-21.6512\end{array}\right], \mathbf{G}_{3}=\left[\begin{array}{ll}222.2539 & 529.3-\end{array}\right.$ 20.7009], $\mathbf{G}_{4}=[525.7506535 .8547-21.0306]$.

It should be noted by applying the given LMIs the sign operator in (17) becomes its inverse.

Hence, the poles of the closed loop at the vertices are: $\lambda\left(\mathbf{A}_{1}+\mathbf{B G}_{1}\right)=[-0.5082-9.5097+1.8531 i-9.5097-$ $1.8531 i]^{\top}, \lambda\left(\mathbf{A}_{2}+\mathbf{B G}_{2}\right)=[-0.3952-9.3790+0.9612 i-$ $9.3790-0.9612 i]^{\top}, \lambda\left(\mathbf{A}_{3}+\mathbf{B G}_{3}\right)=[-1.5562+2.3167 i-$ $1.5562-2.3167 i-4.0906]^{\top}, \lambda\left(\mathbf{A}_{4}+\mathbf{B G}_{4}\right)=[-1.6765+$ $0.8012 i-1.6765-0.8012 i-4.1797]^{\top}$. During operation all of the possibly occurring closed-loop poles lie within the region characterized by the closed-loop poles at the vertices.

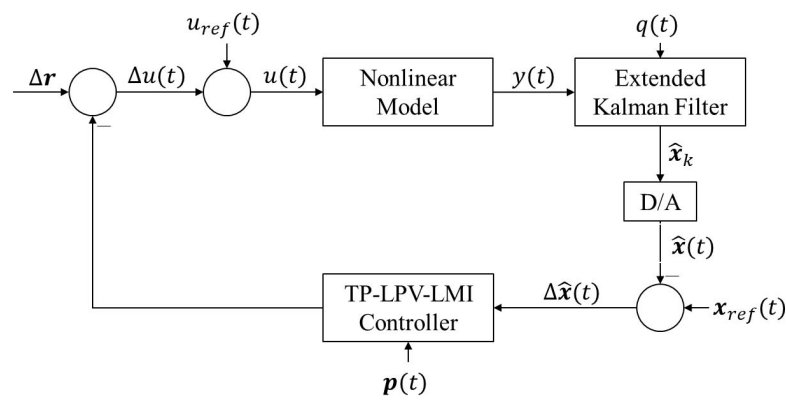

Figure 2. Structure of the control loop.

\section{Final Control Structure}

We have considered the original model as reference model during the controller design by assuming that it is an exactly known model from parameter and dynamics point of view. This model can be replaced by any arbitrary, but appropriate model, however.

The finalized control structure can be seen in Fig. 2. We have applied permanent $u_{\text {ref }}(t)=u_{\text {ref }}=14[\mathrm{mg} / \mathrm{kg} / \mathrm{min}]$ which guaranteed that $x_{1}(t)=1\left[\mathrm{~mm}^{3}\right]$ at the end of the therapy.

Since the $x_{2}$ and $x_{3}$ state variables of the controlled system cannot be measured we applied an Extended Kalman Filter (EKF) to estimate them. The EKF was a mixed continuous/discrete EKF with $T=1$ day sampling time in its measurement part [17], [33].

Through the control framework the controller enforces the original model to behave as the given reference model, namely, $\mathbf{x}(t)=\mathbf{x}_{\text {ref }}(t), t \rightarrow \infty$. This is equivalent to $\Delta \mathbf{r}=\mathbf{x}-$ $\mathbf{x}_{\text {ref }}=\mathbf{0}$, where $\Delta \mathbf{r}=\mathbf{0}$, namely, the controller eliminates the deviation between $\mathbf{x}$ and $\mathbf{x}_{r e f}$ over time.

\section{RESULTS}

The validation of the developed control environment have been realized in the MATLAB Simulink environment. The applied initial conditions have been $\mathbf{x}\left(t_{0}\right)=[\ln (14900), \ln (14900), 0]^{\top}$, $\mathbf{x}_{\text {ref }}\left(t_{0}\right)=[\ln (17000), \ln (17000), 0]^{\top}, \quad \hat{\mathbf{x}}\left(t_{0}\right)=$ $[\ln (17000), \ln (17000), 0]^{\top}, \quad$ respectively. The initial conditions were arbitrarily selected, however, we assumed the lack of therapeutic agents before the beginning of the therapy $\left(x_{3}\left(t_{0}\right)=\hat{x}_{3}\left(t_{0}\right)=x_{r e f, 3}\left(t_{0}\right)=0\right)$ and the $\mathbf{x}_{r e f}\left(t_{0}\right)=\hat{\mathbf{x}}\left(t_{0}\right)$, thus the reference and EKF state variables are adjusted by us at the beginning of the therapy. The selected values were reasonable from the viewpoint of the domain of $\mathbf{x}_{i}$.

We have not considered noises and disturbances in this study in accordance with the properties of the model, i.e. $d \equiv 0$ and $n \equiv 0$. This assumption leaded the EKF to be acting as an optimal estimator [33].

Figure 3 presents the state trajectories of the original system $(\mathbf{x}(t))$, the $\operatorname{EKF}(\hat{\mathbf{x}}(t))$ and the reference system $\mathbf{x}_{r e f}(t)$, 

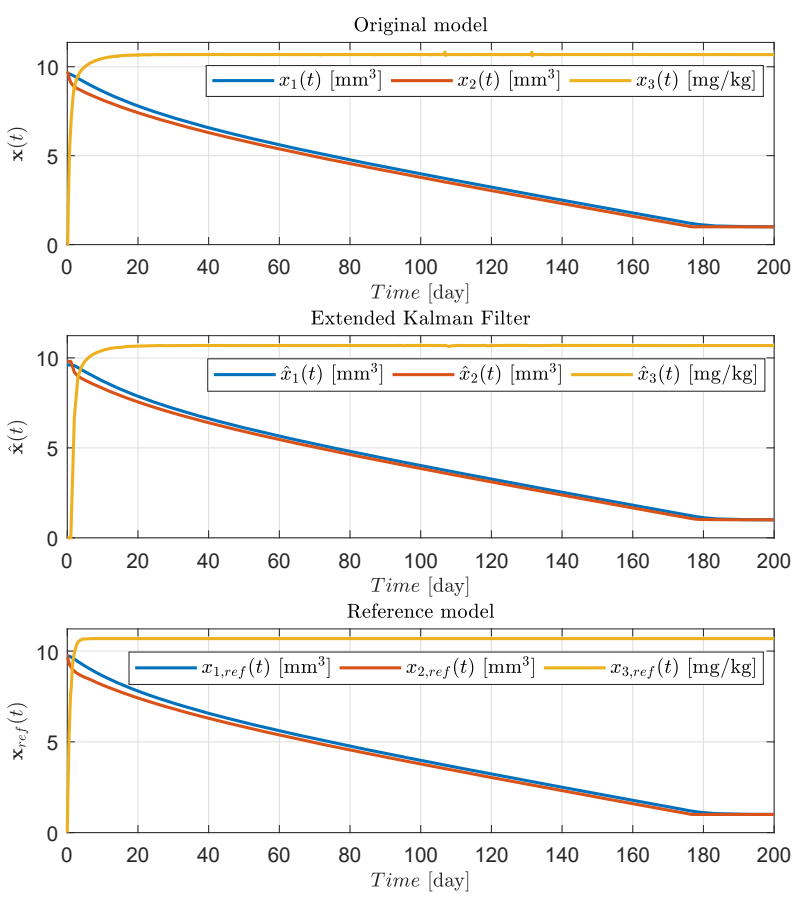

Figure 3. Trajectories of the state variables: $\mathbf{x}(t)$ original system, $\hat{\mathbf{x}}(t) \mathrm{EKF}$, $\mathbf{x}_{\text {ref }}(t)$ reference system.

respectively. It can be seen that both of them are quite similar. The controlled system and the EKF are approaching the reference model with high accuracy. The final values for $x_{1}$ and $x_{2}$ in the case of all models are $1 \mathrm{~mm}^{3}$ which is the lower boundary of the domain of the states as it was expected.

Figure 4 shows the deviation between the state variables of the different systems. The upper subfigure is the difference between the original system and the EKF, namely, $\mathbf{x}(t)-\hat{\mathbf{x}}(t)$. In the closed-loop the EKF approximates the states of the original system. It can be seen that this approximation is loaded with small error in the case of $x_{1}$ and $x_{2}$ compared to the magnitudes of the states, further, this small error decays fast as well. The higher, but quickly decaying error in $x_{3}(t)$ is coming from the discretization error. The middle subfigure is the difference between the reference system and the EKF, that is $\mathbf{x}_{r e f}(t)-\hat{\mathbf{x}}(t)$. From the controller point of view, this is the important aspect, since this is the error signal to be considered by the controller. The same phenomena occurs as in the previous case, namely, the initial errors decay rapidly. The lower subfigure represents the difference between the reference system and the original system, namely, $\mathbf{x}_{r e f}(t)-\mathbf{x}(t)$. The figure shows that the deviations are small, moreover, rapidly decay. The final Root Mean Square Error (RMSE) for all state variables are the following over the whole time domain:

- $\operatorname{RMSE}_{\mathbf{x}(t)-\hat{\mathbf{x}}(t)}=[2.2290,3.8269,3.5293]$;

- $\operatorname{RMSE}_{\mathbf{x}_{r e f}(t)-\hat{\mathbf{x}}(t)}=[2.2116,3.8149,5.4068]$;

- $\operatorname{RMSE}_{\mathbf{x}_{r e f}(t)-\mathbf{x}(t)}=[0.1843,0.0565,2.0559]$.

Consequently, the controller operated well and enforced the controlled original nonlinear system to be behave as the reference system.
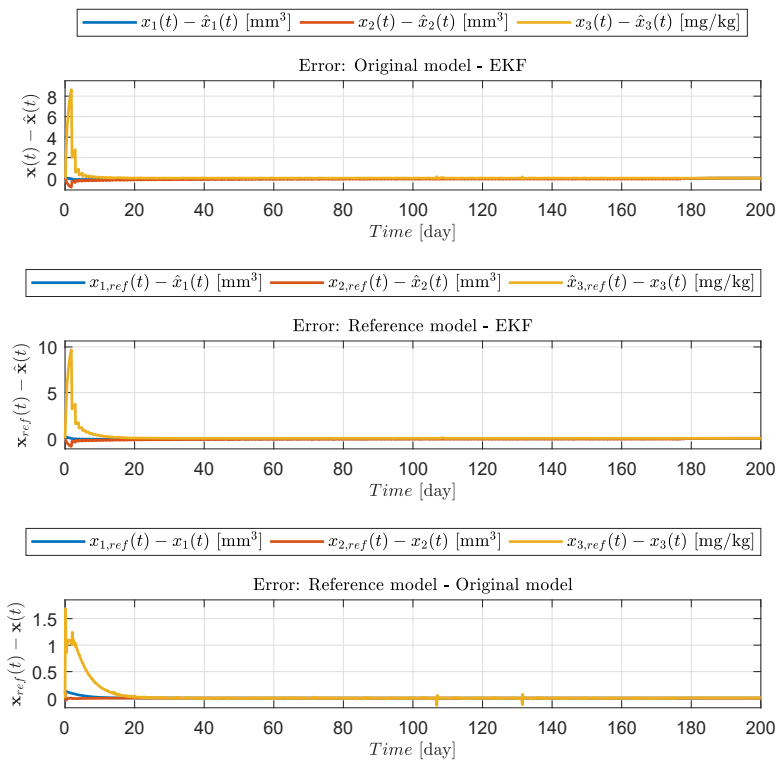

Figure 4. Deviations between the states of the models.
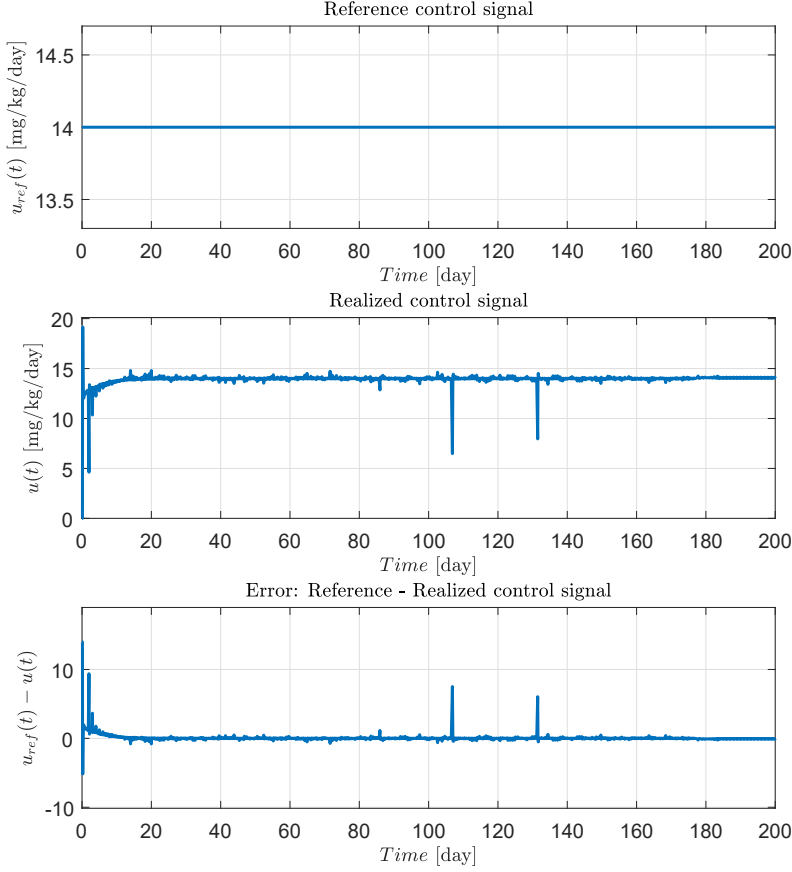

Figure 5. The reference and realized control signals.

Figure 5 shows the reference $u_{r e f}(t)$ and control $u(t)$ 
signals, respectively. Due to the initial value discrepancy there was a higher peak-like difference between the $u_{r e f}(t)$ and $u(t)$ which stabilized rapidly. There are different downward peaks in the $u(t)$ around days 2, 106 and 131 which are caused by the switches in the scheduling parameters. The $u_{\text {ref }}$ was permanent $14 \mathrm{mg} / \mathrm{kg} /$ day as we mentioned already. The RMSE based deviation between $u_{\text {ref }}(t)$ and $u(t)$ was 9.3994 over the whole simulated time horizon. The lower subfigure shows that the controller was able to keep the control signal on similar level as the reference signal, thus the control framework operated well from this point of view as well.

The totally injected reference inhibitor was $U_{\text {ref }}=18298$ $[\mathrm{mg} / \mathrm{kg}]$, while the totally injected inhibitor was $U=18098$ $[\mathrm{mg} / \mathrm{kg}]$ which is only $200[\mathrm{mg} / \mathrm{kg}]$ discrepancy.

\section{CONCLUSION}

In this study we have presented our latest developments regrading the control of tumor growth through anti-angiogenic treatment. We have applied the extended, transformed version of well-known Hahnfeldt-model from which two internal states have been estimated by EKF.

We have developed a difference based qLPV model which has been used via the LMI based controller design. The realization was done by applying the TP model transformation to generate TP controller. The resulting TP-LPV-LMI controller is able to provide stable control action in Lyapunov sense. We have found that the developed control framework operated well and it was able to enforce the controlled model to behave as the determined reference model.

In our future work we will develop more sophisticated reference model and embed the disturbance and noise effects to examine whether the controller can tolerates them or not.

\section{ACKNOWLEDGMENT}

The Authors thankfully acknowledge the support of the Robotics Special College of Óbuda University and the Óbuda University's Research, Innovation and Service Center.

\section{REFERENCES}

[1] N. Vasudev and A. Reynolds, "Anti-angiogenic therapy for cancer: current progress, unresolved questions and future directions," Angiogenesis, vol. 17 , no. 3 , pp. 471-494, 2014.

[2] P. Charlton and J. Spicer, "Targeted therapy in cancer," Medicine, vol. 44, no. 1, pp. 34-38, 2016.

[3] R. Weinberg, The biology of cancer, 2nd ed. New York, USA: Garland Science, Taylor and Francis, 2014

[4] A. M. Abdalla, L. Xiao, M. W. Ullah, M. Yu, C. Ouyang, and G. Yang, "Current challenges of cancer anti-angiogenic therapy and the promise of nanotherapeutics," Theranostics, vol. 8, no. 2, pp. 533-548, 2018

[5] J. Sápi, L. Kovács, D. Drexler, P. Kocsis, D. Gaári, and Z. Sápi, "Tumor volume estimation and quasi-continuous administration for most effective bevacizumab therapy," PLOS ONE, vol. 10, no. 11, 2015.

[6] D. Drexler, J. Sápi, and L. Kovács, "Potential Benefits of Discrete-Time Controller-based Treatments over Protocol-based Cancer Therapies," ACTA Pol Hung, vol. 14, no. 1, pp. 11-23, 2017.

[7] J. Sápi, "Controller-managed automated therapy and tumor growth model identification in the case of antiangiogenic therapy for most effective, individualized treatment," Ph.D. dissertation, Óbuda University, Budapest, Hungary, 2015.

[8] D. A. Drexler, J. Sápi, and L. Kovács, "Positive nonlinear control of tumor growth using angiogenic inhibition," IFAC-PapersOnLine, vol. 50, no. 1, pp. 15068 - 15073, 2017, 20th IFAC World Congress.
[9] A. White, G. Zhu, and J. Choi, Linear Parameter Varying Control for Engineering Applicaitons, 1st ed. London: Springer, 2013.

[10] L. Kovács, "Linear parameter varying (LPV) based robust control of type-I diabetes driven for real patient data," Knowl-Based Syst, vol. 122, pp. 199-213, 2017.

[11] S. Boyd, L. El Ghaoui, E. Feron, and V. Balakrishnan, Linear Matrix Inequalities in System and Control Theory, ser. Studies in Applied Mathematics. Philadelphia, PA: SIAM, 1994, vol. 15.

[12] G. Herrmann, M. C. Turner, and I. Postlethwaite, "Linear matrix inequalities in control," in Mathematical methods for robust and nonlinear control. Springer, 2007, pp. 123-142.

[13] P. Baranyi, Y. Yam, and P. Varlaki, Tensor Product Model Transformation in Polytopic Model-Based Control, 1st ed. USA: CRC Press, 2013.

[14] R.-E. Precup, R.-C. David, and E. M. Petriu, "Grey wolf optimizer algorithm-based tuning of fuzzy control systems with reduced parametric sensitivity," IEEE Transactions on Industrial Electronics, vol. 64, no. 1, pp. 527-534, 2017.

[15] P. Baranyi, "Extension of the Multi-TP Model Transformation to Functions with Different Numbers of Variables," Complexity, vol. 2018, 2018.

[16] D. Copot, R. De Keyser, J. Juchem, and C. Ionescu, "Fractional order impedance model to estimate glucose concentration: in vitro analysis," ACTA Pol Hung, vol. 14, no. 1, pp. 207-220, 2017.

[17] M. Grewal and A. Andrews, Kalman Filtering: Theory and Practice Using MATLAB, 3rd ed. Chichester, UK: John Wiley and Sons, 2008.

[18] P. Hahnfeldt, D. Panigrahy, J. Folkman, and L. Hlatky, "Tumor development under angiogenic signaling: A dynamical theory of tumor growth, treatment response, and postvascular dormancy," Cancer Res, vol. 59, pp. 4770-4775, 1999.

[19] J. Klamka, H. Maurer, and A. Swierniak, "Local controllability and optimal control for amodel of combined anticancer therapy with control delays," Math Biosci Eng, vol. 14, no. 1, pp. 195-216, 2017.

[20] J. Sápi, D. A. Drexler, and L. Kovács, "Potential Benefits of DiscreteTime Controllerbased Treatments over Protocol-based Cancer Therapies," Acta Pol Hung, vol. 14, no. 1, pp. 11-23, 2017.

[21] J. Mohammadpour and C. W. Scherer, Control of linear parameter varying systems with applications. Springer Science \& Business Media, 2012.

[22] J. Kuti, P. Galambos, and P. Baranyi, "Control analysis and synthesis through polytopic tensor product model: a general concept," IFACPapersOnLine, vol. 50, no. 1, pp. 6558-6563, 2017.

[23] G. B. Thomas, R. L. Finney, M. D. Weir, and F. R. Giordano, Thomas' calculus. Addison-Wesley Reading, 2003.

[24] J. Kuti, P. Galambos, and P. Baranyi, "Minimal volume simplex (MVS) convex hull generation and manipulation methodology for TP model transformation," Asian J Control, vol. 19, no. 1, pp. 289-301, 2017.

[25] — - "Generalization of tensor product model transformation for control design," IFAC-PapersOnLine, vol. 50, no. 1, pp. 5604-5609, 2017.

[26] A. Szollosi and P. Baranyi, "Influence of the Tensor Product Model Representation of qLPV Models on the Feasibility of Linear Matrix Inequality Based Stability Analysis," Asian J Control, vol. 20, no. 1, pp. 531-547, 2018.

[27] X. Liu, Y. Yu, Z. Li, H. H. Iu, and T. Fernando, "An Efficient Algorithm for Optimally Reshaping the TP Model Transformation," IEEE T Circuits-II, vol. 64, no. 10, pp. 1187-1191, 2017.

[28] P. G. M. Chilali and P. Apkarian, "Robust Pole Placement in LMI Regions," IEEE T Automat Contr, vol. 44, no. 12, pp. 2257 - 2270, 1999.

[29] M. Chilali and P. Gahinet, " $\mathrm{H}_{\infty}$ Design with Pole Placement Constraints: An LMI Approach," IEEE Transactions on Automatic Control," IEEE T Automat Contr, vol. 41, no. 3, pp. 358 - 367, 1996.

[30] K. Tanaka and H. O. Wang, Fuzzy Control Systems Design and Analysis: A Linear Matrix Inequality Approach, 1st ed. Chichester, UK: John Wiley and Sons, 2001.

[31] J. Löfberg, "Yalmip : A toolbox for modeling and optimization in matlab," in Proceedings of the CACSD Conference, Taipei, Taiwan, 2004.

[32] I. Polik, T. Terlaky, and Y. Zinchenko, "Sedumi: a package for conic optimization," in IMA workshop on Optimization and Control, Univ. Minnesota, Minneapolis. Citeseer, 2007.

[33] H. Musoff and P. Zarchan, Fundamentals of Kalman Filtering: A Practical Approach, 3rd ed. American Institute of Aeronautics and Astronautics, 2009. 